\title{
Addendum: Vibrational spectroscopic image analysis of biological material using multivariate curve resolution-alternating least squares (MCR-ALS)
}

Judith Felten, Hardy Hall, Joaquim Jaumot, Romà Tauler, Anna de Juan and András Gorzsás

Addendum to: Nature Protocols https://doi.org/10.1038/nprot.2015.008, published online 8 January 2015

The GUI described in this paper has undergone extensive expansion since its original publication. While the core concept and workflow remain unchanged, new functions have been added to each main part. The pre-processing part of the GUI now includes controls to trim the spectral region, to perform multiple forms of normalization and to perform quick intensity evaluations (integration or single-point intensity evaluation), as well as an option to save and load pre-processing parameters. The most significant changes in the MCR-ALS part of the GUI include different ways of setting starting points (initial estimates) for the iterations, as well as the capability to use additional constraints, such as equality and unimodality. The final third of the GUI now includes options to manually select, label and export spectra from the dataset; change visualization (changing color schemes, changing dimensions via refolding and changing apparent resolution via oversampling); and utilize spectra loaded as references or manually selected as initial estimates for MCR-ALS.

In addition to these expansions, we made the code itself more effective by adapting it to the latest version of MATLAB. Special care was taken to maintain compatibility with MATLAB versions at least as old as 2014a. Taking advantage of newer built-in MATLAB functions, the GUI is now able to read Microsoft Excel .xls(x) files both for the original dataset and for reference spectra.

The updated GUI, including a manual (detailing the listed changes with step-by-step explanations and screenshots) and demo datasets as tutorials (in .mat, .txt and .xlsx format), is available at https://www.umu.se/en/research/infrastructure/visp/downloads/. Please click on "Latest stable version of the MCR-ALS GUI, including manual and demo dataset" to download the latest version of the GUI as a .zip archive.

Published online: 5 June 2019

https://doi.org/10.1038/s41596-019-0196-9 